FERMILAB-TM-1904

\title{
Report III on Switchyard Beam Position Monitor
}

\author{
Hengjie Ma \\ Fermi National Accelerator Laboratory \\ P.O. Box 500, Batavia, Illinois 60510
}

September 1994 


\section{Disclaimer}

This report was prepared as an account of work sponsored by an agency of the United States Government. Neither the United States Government nor any agency thereof, nor any of their employees, makes any warranty, express or implied, or assumes any legal liability or responsibility for the accuracy, completeness, or usefulness of any information, apparatus, product, or process disclosed, or represents that its use would not infringe privately owned rights. Reference herein to any specific commercial product, process, or service by trade name, trademark, manufacturer, or otherwise, does not necessarily constitute or imply its endorsement, recommendation, or favoring by the United States Government or any agency thereof. The views and opinions of authors expressed herein do not necessarily state or reflect those of the United States Government or any agency thereof. 


\title{
Report III on Switchyard Beam Position Monitor
}

\author{
Hengjie Ma \\ January, 1993
}

\section{SUMMARY}

To detect the extremely weak signal of a Switchyard bpm, the switchyard beam position monitor (SY_BPM) must have very high gain. To obtain smooth measurement data from the signal with poor SNR, the bpm must also have a very narrow bandwidth. However, maintaining the gain balance and particularly the phase balance between the two channels of the bpm has been found difficult. The instability of channel balance has affected the accuracy and validity of the measurement. The bpm currently used in Switchyard was intended for the medium beam intensity range of about 1E12 protons/20s. Its circuitry design does not have much potential for increased sensitivity. To extend its capability of handling beams with lower intensity, the gain of the bpm has to be further raised, and its bandwidth also has to be further narrowed. That will inevitably make the situation even worse. To solve the problem of channel imbalance, an experimental prototype of the SY_BPM was designed and fabricated in June, 1992. On the prototype, the processing of LOG conversion, instead of AM/PM conversion, was used to make the system less affected by the phase imbalance, and a multiplexed channel was designed to avoid channel mismatch problem.

The prototype was systematically tested and compared against our current bpm. The test results show that the design of the first prototype is basically successful. However, it also reveals some flaws with the processing scheme of LOG conversion which the prototype design is based upon. Briefly, there are two major flaws. One is the LOG conversion nonlinearity of the analog IC chip used, which has limited the effective measurement range of beam displacement. Another flaw is the sensitivity of the LOG conversion accuracy to noise, which has limited the lower bound of the beam intensity which the bpm can handle.

Over the past six months, careful study and analysis of the first prototype as well as other bpm designs were conducted. With the gained knowledge, we have designed the second experimental SY_BPM prototype. This prototype combines the advantages of good channel balance with the multiplexed channel scheme and the advantages of wide dynamic range of AM/PM conversion scheme. So far, the major part of the circuitry 
design has been completed. The result of circuits verification looks promising. The fabrication can be expected to start in February, 1993.

In the following sections, we will first discuss some basic beam position measurement schemes. Then, we will analyze the characteristics of several possible bpm designs to find out the optimum scheme for the switchyard application. Finally, the design of the second SY_BPM prototype generated from our research will be introduced. As a byproduct of developing the second prototype, a possible design of the bpm for handling fast spill with very short duty-cycle has also been found.

\section{BASIC DESIGN SCHEMES OF COMMONLY USED BPMS}

The bpms currently used were developed about two decades ago. The designs which are being widely used can be categorized into only two basic schemes; AM-to-PM conversion and direct amplitude comparison scheme.

\section{AM/PM scheme}

Figure 1-(a) shows the simplified block diagram of a typical bpm system using the AM/PM conversion scheme. In this system, the beam detector outputs two in-phase signals. The ratio of their amplitudes, $\mathrm{A}$ over $\mathrm{B}$, is a monotone function of beam position. The signals are first processed by a amplitude modulation-to-phase modulation (AM/PM) converter consisting of a $90^{\circ}$ phase shifter and a $180^{\circ}$ hybrid junction. After the conversion, the two original in-phase signals with unequal amplitudes are converted into two out-of-phase signals with equal amplitudes. The phase difference varies from 0 to $180^{\circ}$ corresponding to beam position. Since the signal amplitude no longer carries any information after the conversion, two amplitude limiters are used to clip the amplitudes into a fixed value to prevent intensity variation from affecting position measurement. The phase difference is detected by a multiplier ${ }^{1}$. A lowpass filter smooths out the output of the phase detector. The position measurement is finally obtained by scaling the output.

$\mathrm{AM} / \mathrm{PM}$ scheme is relatively simple. One of the advantages which the scheme has is its very large input dynamic range. That is because of the use of the amplitude limiters. The limiters make the system always work under a saturating condition. The AM/PM converter used consists of two linear passive devices. Therefore, it can handle large

1 For low frequencies, an XNOR gate may be used for the phase detection. 


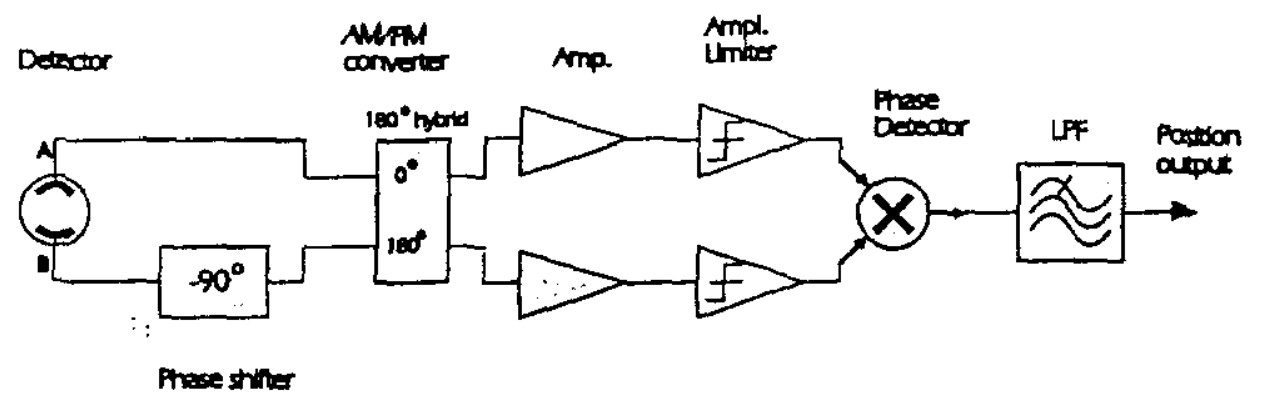

(a)

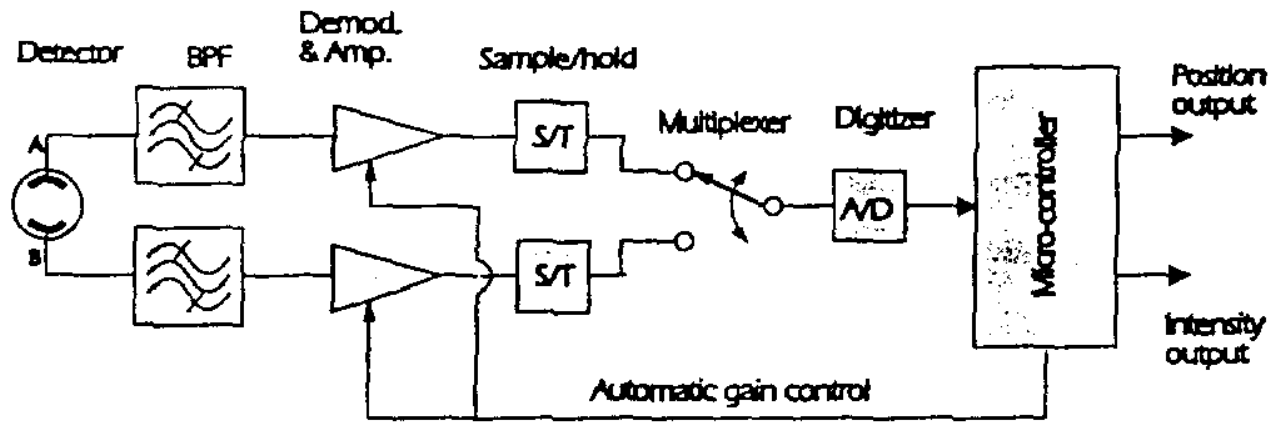

(b)

Figure 1. Two design shemes of commonly used beam position monitors 
beam displacement. That is another advantage with AM/PM scheme.

However, there is a practical problem with this scheme. For the channel section from the beam detector to the phase detector, both gain and phase have to be balanced. For the channel section after the converter, the phase balance must be maintained. Otherwise, the center of position measurement will drift off zero. There would also be a problem if a quarter-wavelength delay-line was used as the $90^{\circ}$ phase shifter. Because of this delayline, the two signals from the beam detector cannot arrive at the AM/PM converter at the same time. That would create measurement error when the width of a fast-spill is very narrow.

\section{Direct amplitude comparison scheme}

The idea of directly comparing the amplitudes of two signals is straightforward. Figure 1-(b) shows the scheme based upon such an idea. The signals from plate A and B are filtered and linearly amplified. The amplitudes of the signals are obtained with a certain type of rectifier, and then digitized. A micro-processor calculates the ration of the two amplitudes to determine beam position. It also calculates the average of the amplitude to determine beam intensity. The advantage with this scheme is that the signal processing is very simple. The disadvantage is that its input dynamic range is limited since its amplifiers have to work within their linear region, and the digitizer requires adequate signal level in order to keep the quantization error below a certain level. Certainly, adding an automatic gain control (AGC) circuit may relieve this problem. However, the AGC needs a certain amount of time to respond to a sudden change in signal level. This makes it unsuitable to short spill applications.

Besides these schemes, a scheme of using analog log-amp IC to perform the position measurement has been proposed. The idea is to use a log-amplifier on each channel. The two log-amplifiers output the log-amplitudes of the two signals respectively. Since the division operation in linear domain converts into a subtraction operation in logarithm domain, the amplitude ratio of the two signals can be obtained with a differential amplifier. Figure 2-(a) shows such a scheme. The limitation with this scheme is that it requires that the input signals have a good signal-to-noise ratio. Otherwise, the conversion accuracy would degrade. Another problem is the nonlinear error associated with the analog devices. The nonlinearity problem has limited the range of beam displacement which the scheme can handle. At present time, it seems that this scheme is still in the study phase. 
Figure 2-(b) shows the design of the first experimental prototype of SY_BPM. This design is based upon the log-amp scheme. In this design, a multiplexed channel is used to eliminate the possible problem of unbalanced signal processing on the two signals. The tests conducted on this prototype enabled us to evaluate the applicability of the logamp scheme, and gain some first-hand experience.

\section{OPTIMUM BPM SCHEME FOR SWITCHYARD APPLICATION}

The major challenge to a BPM for Switchyard application is the extremely low signal level and very poor SNR. So far, our bpm upgrading work has focused only on the improvement of the electronics part. As far as the basic design scheme is concerned, the

choice is only among the three schemes shown in Figure 1 and Figure 2. For the logamp scheme shown in Figure 2-(a), besides the nonlinearity problem, we also found its accuracy too sensitive to the noise in signal based on our test results. That has made it not the best choice for Switchyard because our signals never have a good SNR. The direct comparison scheme shown in Figure 1-(b) does not seem to be a good candidate either because of its limited input dynamic range. In this case, it seems that the answer may be found only from the old AM/PM scheme shown in Figure 1-(a).

The conventional AM/PM scheme typically suffers center offset due to channel imbalance and transient error due to the delay-line used in it. To make it suitable for the Switchyard bpm to be upgraded, the basic design has to be improved. A BPM based on AM/PM scheme comprises four basic blocks; the beam detector which is the source of the original signals, the AM/PM converter consisting of phase shifter and hybrid junctions, the two channels for transmitting and processing signals, and a multiplier with two limiters as the phase detector. Here, the so called "channel" refers to the cables transmitting the signals from the beam detector in the tunnel to the electronics in the service building and the electronics processing the signals. For the system configuration, the arrangement and design of the blocks may vary except that the beam detector has to be the first stage and the phase detector has to be the last stage. Figure 3-(a)through (e) show five possible variants of basic AM/PM scheme. In following sections, we will analyze the performance of each variant in terms of their sensitivity of measurement accuracy to channel mismatch. 


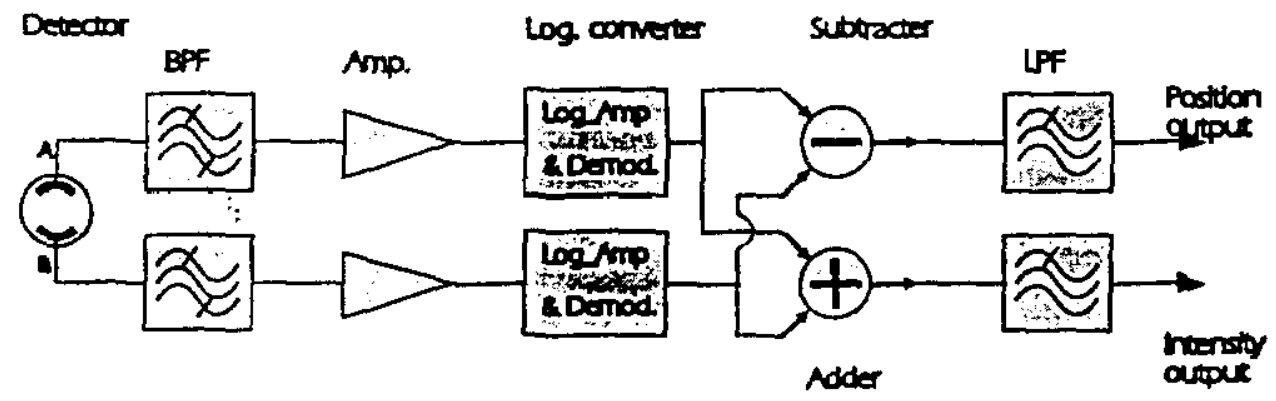

(a)

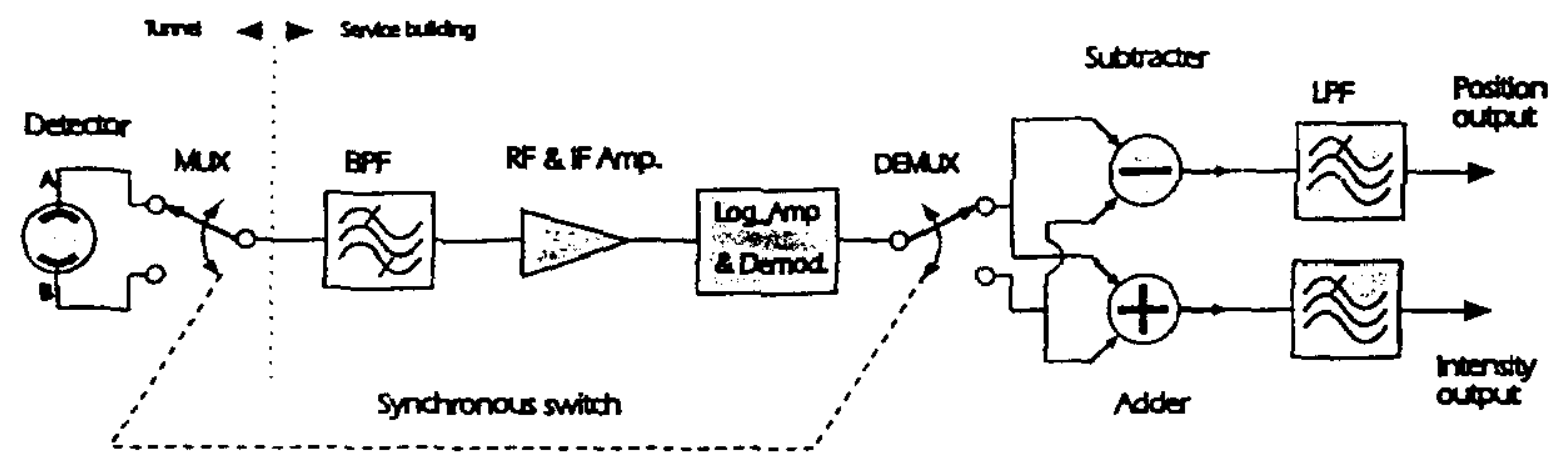

(b)

Figure 2. (a) Proposed BPM design scheme using Log-amp IC. Its applicability is atill under study. (b) Design scheme of 1st SY_BPM prototype. This design uses a multiplerced channel to avoid channel mismatch. 

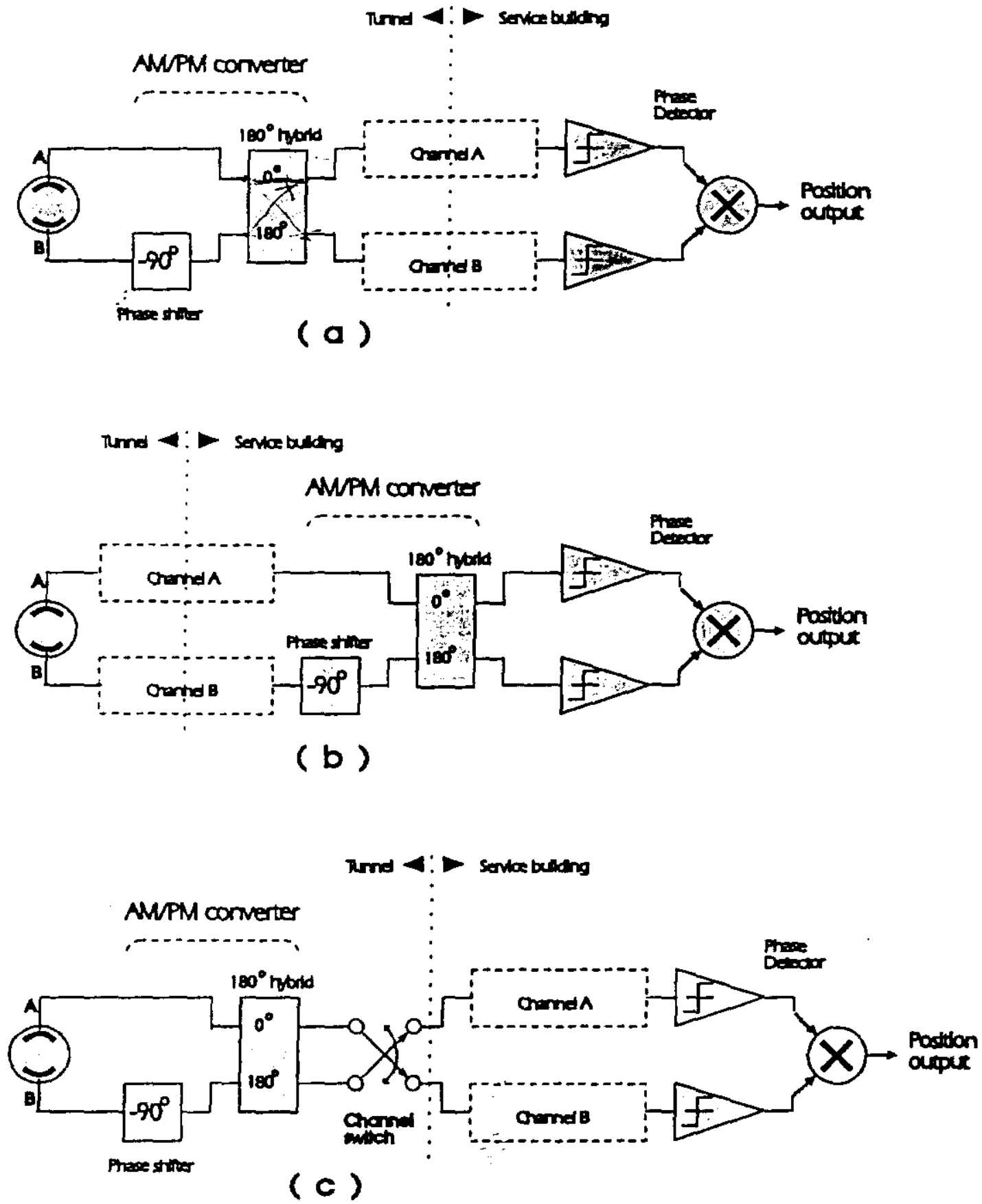

Figure 3. Five possible variants of AM/PM scheme. (a) AM/PM converter on front-end, (b) AM/PM converter on rear-end, (c) The converter on front-end and with a chamel switch. 

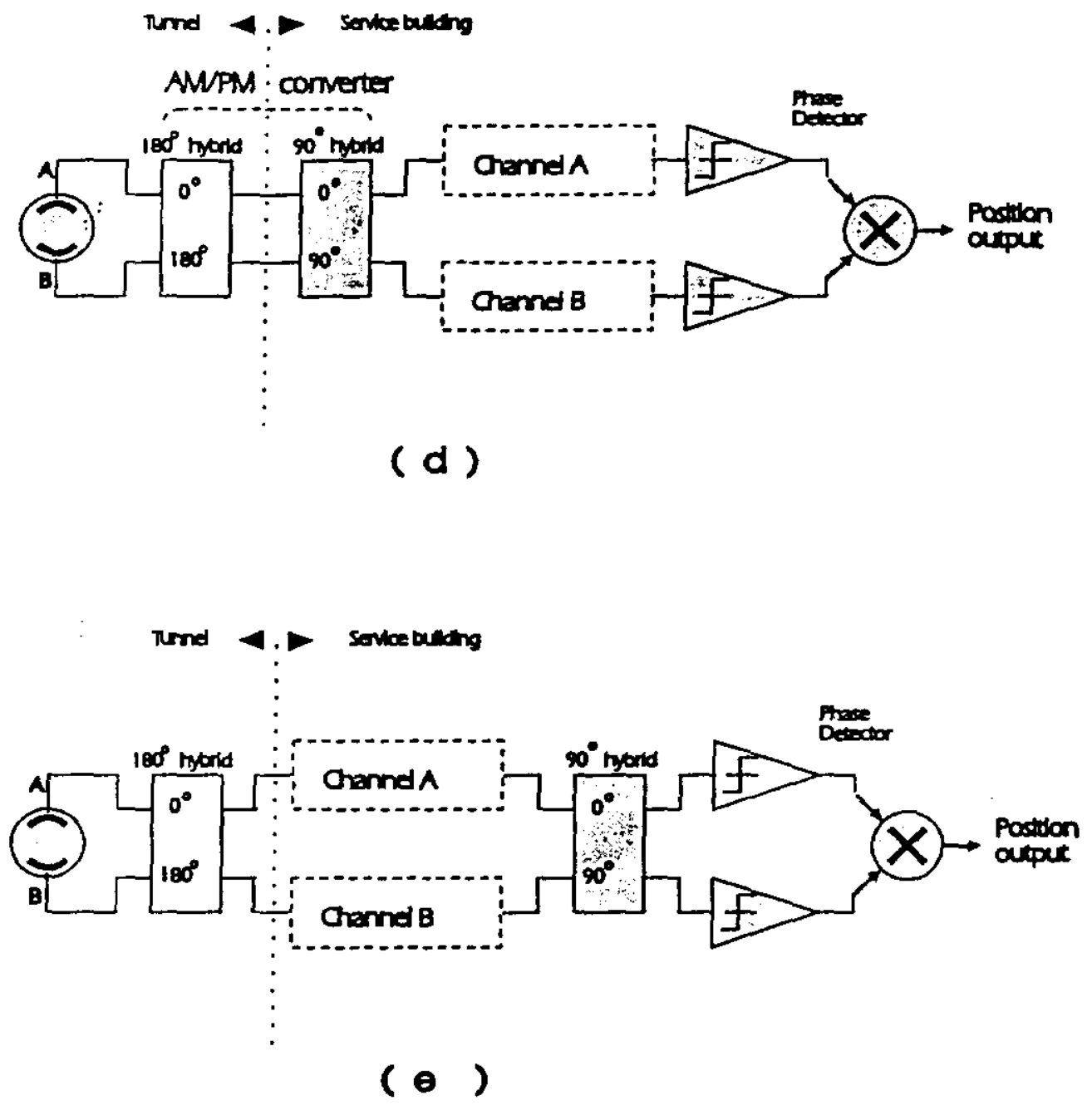

Figure 3. (d) A newly proposed scheme. The AM/PM converter used does not have so called "transient time" proble. (e) Modified design (d). 


\section{Configuration (a)}

The configuration shown in Figure 3-a is the original form of AM/PM system. In this design, the AM/PM converter is located right at the beam detector in the tunnel. Therefore, the detector signals get converted before being sent to electronics through cables. Since after the conversion only the phase difference of the two converted signals carries the position information, the gain mismatch of the channels will not affect measurement accuracy. In other words, only the phase mismatch of the channels can cause measurement error. Assuming that the detector sensitivity to beam displacement is

$0.67 \mathrm{x}=20 \log (\mathrm{A} / \mathrm{B})$,

The error curves are plotted for the case that the beam displacement $x=-10,-5,0$, +5 and $+10 \mathrm{~mm}$ respectively, where $\mathbf{x}$ is the beam displacement, $\mathrm{A}$ and $\mathrm{B}$ are the amplitudes of two plate signals. The position measurement error of this configuration associated with the phase mismatch of channels is plotted in Figure 4-a. It can be seen that the measurement error with this design is very sensitive to the phase mismatch no matter where the beam is located 2 . It means that small changes in the channel phase balance can cause the offset of measurement center.

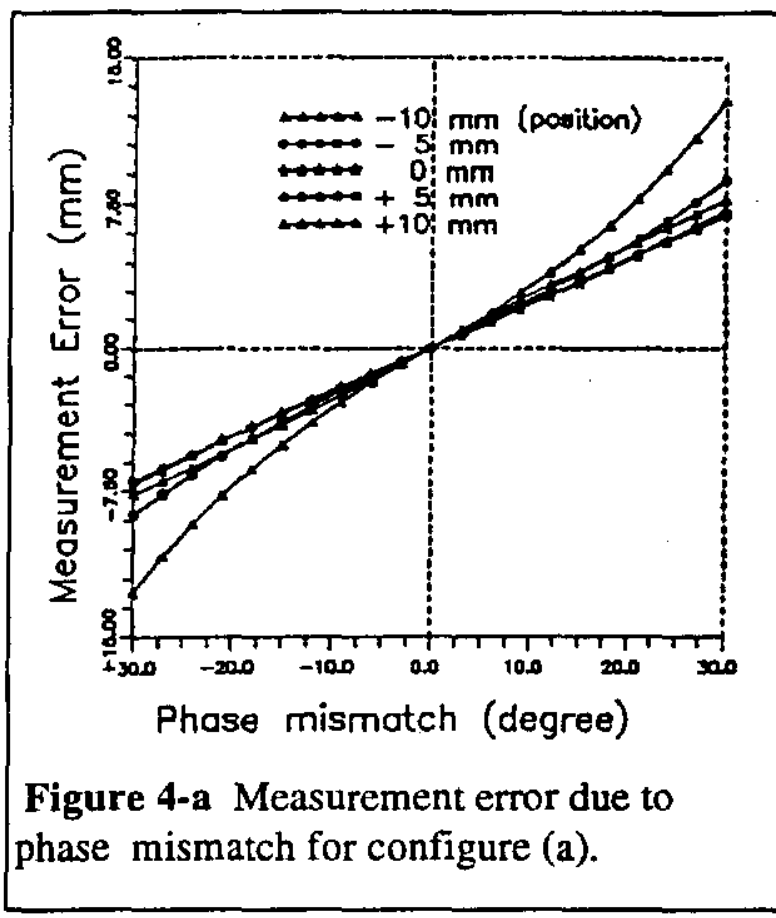

\section{Configuration (b)}

In contrast to design (a), the design shown in Figure 3-(b) moves the AM/PM converter behind the channels. That is, the signals from the detector plates are first sent out of the tunnel and processed without any manipulation. The AM/PM conversion is the last step in the processing before the phase detection. Since the signal amplitudes before the conversion carry the position information, it can be expected that both the gain 2 The scale of the vertical axis for this chart is three time larger than the others. 
and phase imbalance of the channels may affect the measurement accuracy. With the same procedure, the error associated with the phase and gain mismatch are plotted in Figure 4-b-1 and 4-b-2 respectively. From Figure 4-b-1, we can see that the phase mismatch of configuration (b) has less effect on measurement accuracy than that of configuration (a) does (please notice that the vertical scale for this figure is only onethird of that for Figure 4-a). Furthermore, the phase mismatch will not cause any error when beam is located at the center, which is indicated by the flat curve for $x=0.0 \mathrm{~mm}$. The trade-off with this design is that the channel gain imbalance also contributes error, and may causes center offset.
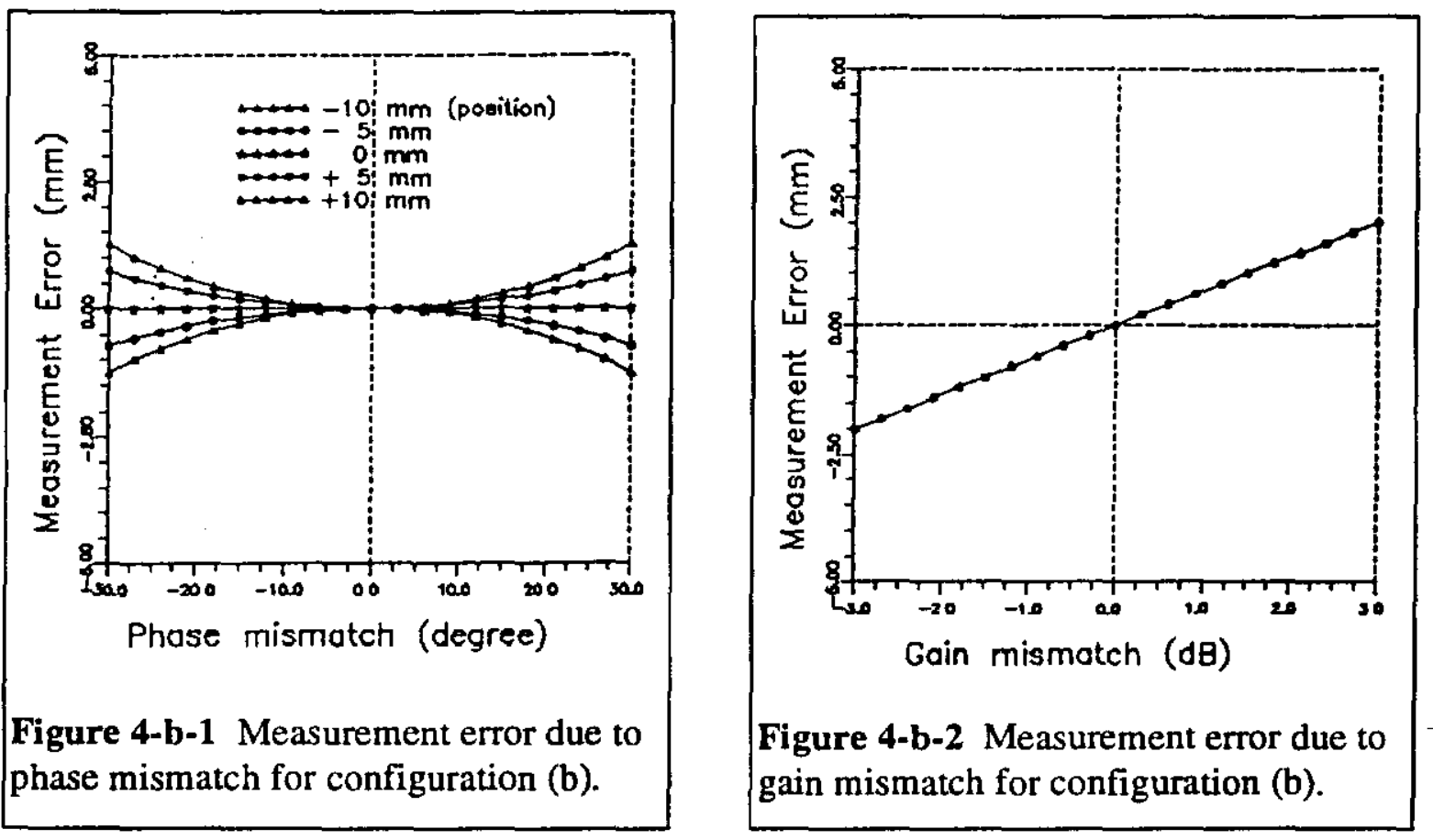

\section{Configuration (c)}

Comparing configuration (a) with (b), we have seen that configuration (a) has-only one factor, which is the phase, to affect its accuracy, while configuration (b) has two factors, the phase and gain, to create its measurement error. As far as making improvement is concerned, configuration (a) seems to be simpler to work with. For configuration (a), what we need to find is a way of compensating the error due to the phase mismatch.

Based on such an idea, we have designed the configuration shown in Figure 3-(c). 
Configuration (c) is similar to configuration (a) except that there is a channel switch inserted between $\mathrm{AM} / \mathrm{PM}$ converter and the channels. In this design, the signal from the upper port of $A M / P M$ converter is sent to channel $A$, and the signal from the lower port to channel $B$ when the channel switch is in normal position. When the switch is in reverse position, the signal from the upper port will be sent to channel $B$, while the signal from the lower port to channel $\mathrm{A}$. The purpose of adding such a switch to swap the two channels is to cancel the error caused by the phase mismatch. Now, let us explain a little bit about how it works. Suppose that a certain mount of beam displacement creates a phase difference _ between the signals at the two output ports of AM/PM converter. Let us assume that the signal of the upper port has a phase advance _ over the signal of the lower port. For a triangle phase detector as the one we have used here, the output voltage of the phase detector is proportional to the absolute of the phase difference between its two input signals

$$
\mathrm{V}_{\text {output }}=\mathrm{C}(\pi-|\theta|)
$$

where $\mathrm{C}$ is a scaling constant.

Thus, when the channel phase is matched, the phase detector output for the case of the channel switch in normal position should be

$$
\mathrm{V}_{\text {match }}=\mathrm{C}(\pi-|+\theta|)=\mathrm{C}(\pi-|\theta|)
$$

When the channel phase becomes mismatched for some reason and the mismatch is $+\Delta \theta$, the total phase difference of the two signals which the phase detector receives will be the original phase difference $\theta$ plus the additional phase shift $\Delta \theta$ caused by the channel imbalance. Thus, the phase detector output for the normal switch position would be

$$
\mathrm{V}_{\text {normal channel }}=C(\pi-|+\theta+\Delta \theta|)=C(\pi-\theta-\Delta \theta)
$$

After the channel switch turns to the reverse position, the original phase difference of $+\theta$ will become $-\theta$, and the total phase difference which the phase detector will receive is $-\theta+\Delta \theta$. Thus, the phase detector output for this case will be

$$
\mathrm{V}_{\text {reverted channel }}=\mathrm{C}(\pi-|+\bar{\theta} \cdot \Delta \theta|)=C(\pi-\theta+\Delta \theta)
$$

Since the electronic switch changes its positions at a certain frequency, the averaged phase detector output would be

$$
\mathrm{V}_{\text {ave }}=\left(\mathrm{V}_{\text {normal channel }}+\mathrm{V}_{\text {reverted channel }}\right) / 2=\mathrm{C}(\pi-\theta)=\mathrm{V}_{\text {match }}(5) \text {, }
$$

which is the same as that for the case of matched channels. In the above derivation, it was assumed that the phase mismatch $\Delta \theta<\theta$. The center value of $\theta$ is $90^{\circ}$. The average of the final output is smoothed out by a lowpass filter. 

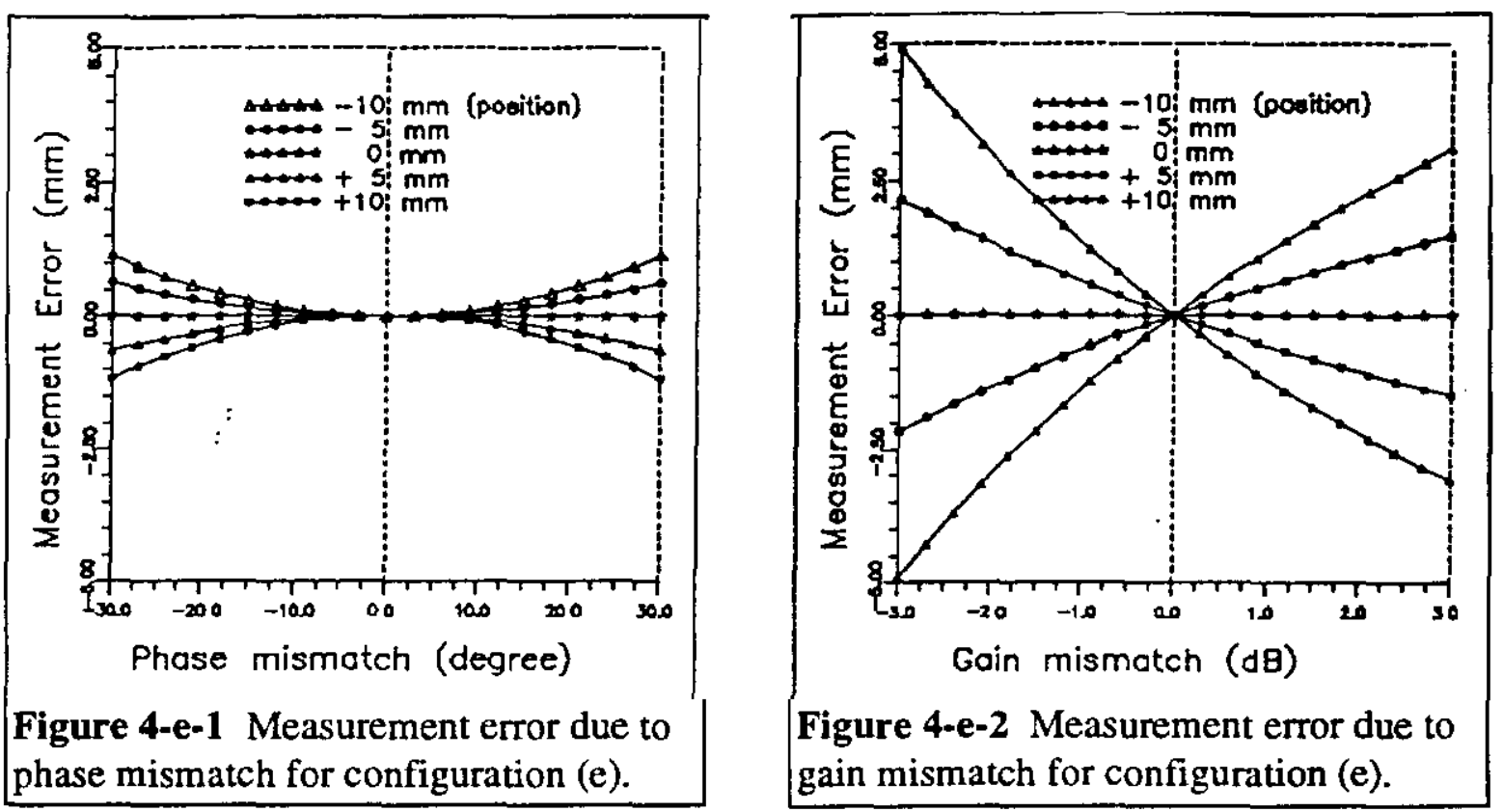

Since the channel bandwidth has to be very narrow in order to suppress noise, the switching frequency of the channel switch cannot be very high. Otherwise, the channel response may be unable to catch up with the switching speed. This might limit its capability of handling very short fast-spill.

\section{Configuration (d.e)}

As it is well known, the conventional AM/PM converter has a so-called transient time problem because of the delay line used as a phase shifter used on it. To avoid this problem, a new design of AM/PM converter without using a delay line has been proposed 3 . The new converter uses one $180^{\circ}$ hybrid cascaded with a $90^{\circ}$ hybrid to complete the AM-to-PM conversion. Getting rid of delay line will eliminate the transient time problem with the converter. The system using such a converter is shown in Figure 3-(d). It was suggested that the first hybrid be placed in the tunnel, while the second one placed at the front end of electronics. By doing so, the error caused by the mismatch of transmission cables between the beam detector and the electronics can be reduced. If it is true that such an arrangement may help to reduce the error due to cable mismatch, the error due to the electronics channel mismatch should also be able to be reduced by simply relocating the second hybrid. Specifically, the second hybrid junction

3 E. Kahana, et al, "Overview of Charged-particle beam diagnostics for the Advanced Photon Source (APS), " paper for 1992 International Workshop on Instrumentation, LBL, 1992. 
may be moved from the front-end to the rear-end of electronics channels, so that the electronics channels become part of the transmission path between the two hybrids. Based upon such an idea, we have designed an improved configuration shown in Figure 3-(e). To evaluate this design, we calculated its measurement errors associated with the gain and phase mismatch of the channels. The results are plotted in Figure 4-e-1 and 4-e2 respectively. We can see that its phase characteristics is very similar to that of the configuration (b). The gain mismatch also causes error on this configuration. However, the interesting point of the result is that both error characteristics curves are symmetric with respect to their horizontal axes, and both have a flat curve for the case $x=0.0 \mathrm{~mm}$. That means both phase and gain mismatch do not cause center offset. The mismatches may only cause scale error when the beam is not at center. This is an important advantage we have found with this design.

\section{THE DESIGN OF THE SECOND SY_BPM PROTOTYPE}

After we analyzed each of the possible designs, it has become clear that the scheme shown in Figure 3-(c) may be the best choice for Switchyard at the present time. Base upon this scheme, a design for the second SY_BPM prototype has been completed, as shown in Figure 5-(a). In this design, two SPDT solid-state switches are used for channel switching. The switching frequency is set to be $500 \mathrm{~Hz}$, which can be changed when needed. Two low noise RF pre-amplifiers will be installed in the tunnel in order to boost the extremely weak signals before they are sent out. The purpose is to improve the SNR of the signals at input end of electronics. Since total gain of the linear portion of the system (before the amplitude limiting) is as high as $140 \mathrm{~dB}$, a single-frequency amplifier with such high gain will inevitably be unstable. To avoid the instability problem, the RF signal of $53.104 \mathrm{MHz}$ is down converted to $455 \mathrm{KHz}$ IF after it gets 40 $\mathrm{dB}$ of gain. The IF strip on each channel provides the system with $100 \mathrm{~dB}$ gain. Its $\mathrm{dB}$ amplitude output is used as a beam intensìty indicator. As mentioned earlier, the circuitry design of this prototype has been completed. The fabrication may start as early as February.

Figure 5-(b) shows another design based upon the scheme in Figure 3-(e). This design uses the new AM/PM converter. Therefore, it may better handle very short fastspill. This design is still under study. 


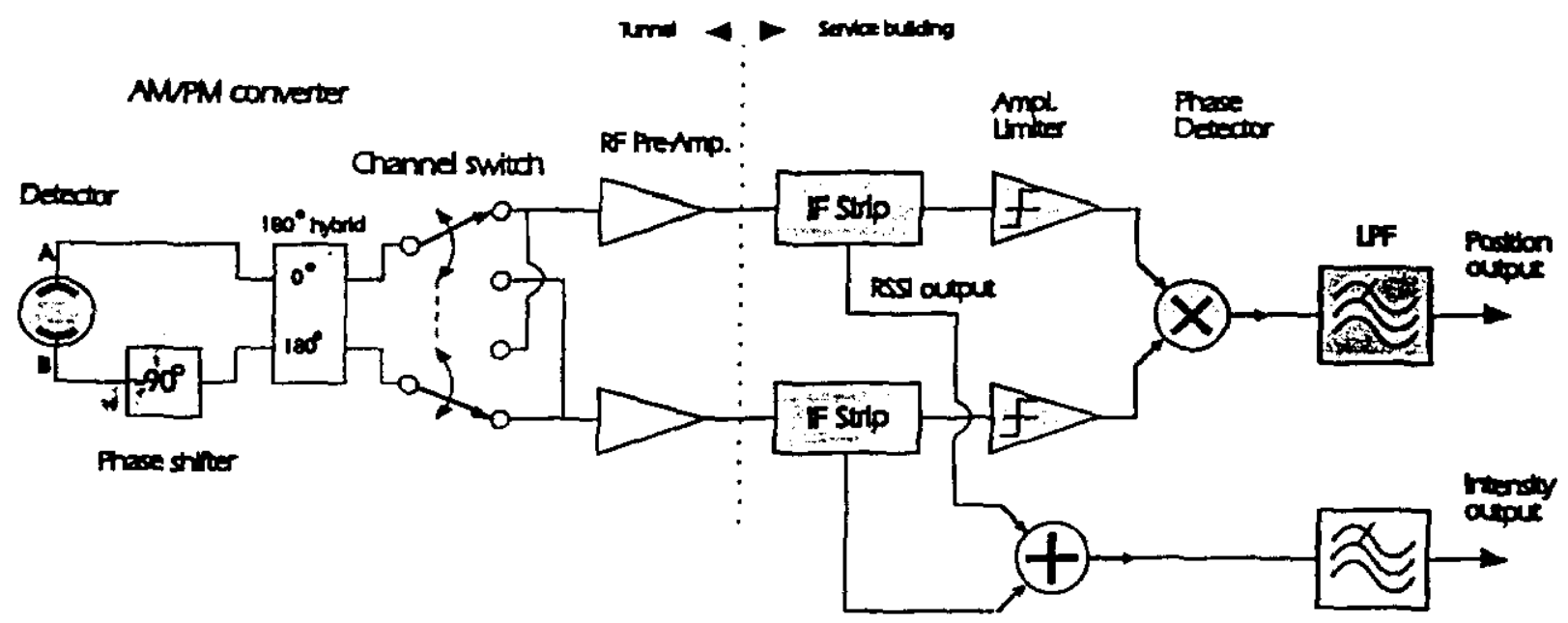

(a)

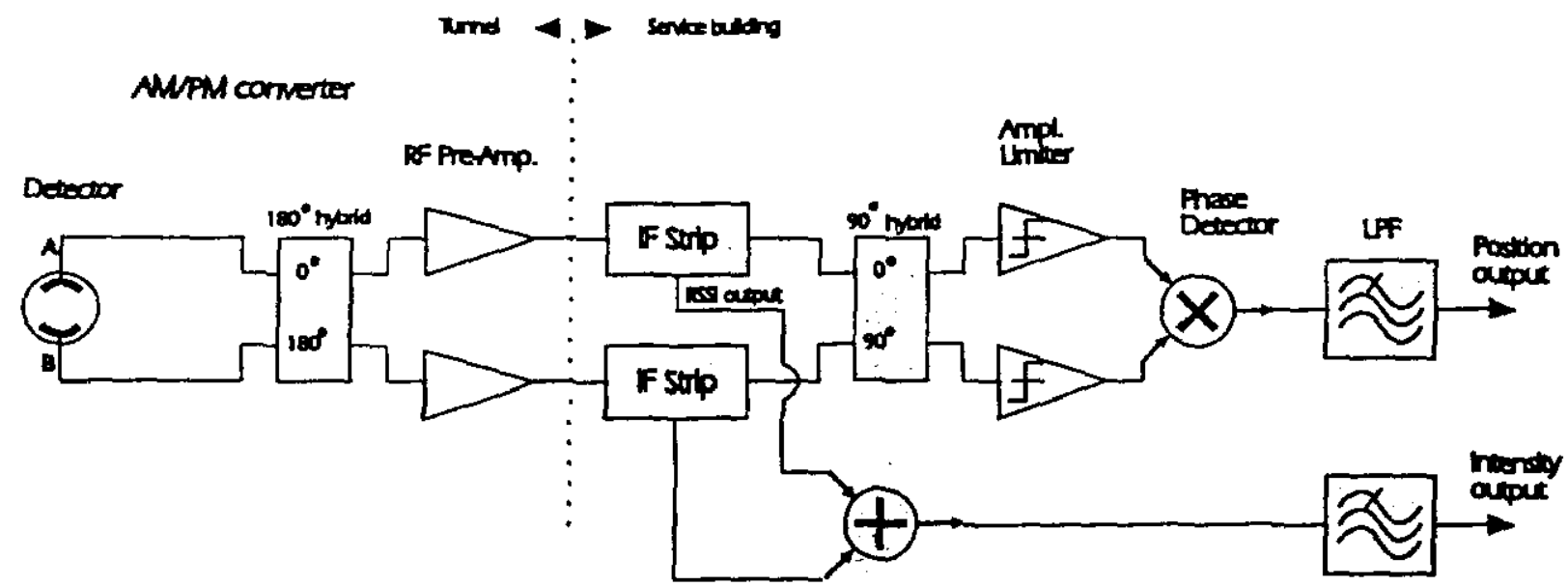

(b)

Figure 5. (a) Design of 2nd SY_BPM prototype. This design uses a channel switch to cancel the cror due to possible channel imbalance. (b) A possible SY_BPM design for better handling very short fast-spills. 